\title{
IL-1 $\beta$ and IL-6 excite neurons and suppress nicotinic and noradrenergic neurotransmission in guinea pig enteric nervous system
}

\author{
Yun Xia, Hong-Zhen Hu, Sumei Liu, Jun Ren, Dimiter H. Zafirov, and Jackie D. Wood \\ Department of Physiology, The Ohio State University College of Medicine and Public Health, Columbus, Ohio 43210, USA \\ Address correspondence to: Jackie D. Wood, Department of Physiology, The Ohio State University College of Medicine and \\ Public Health, 302 Hamilton Hall, 1645 Neil Avenue, Columbus, Ohio 43210, USA. Phone: (614) 292-5449; \\ Fax: (614) 292-4888; E-mail: wood.13.osu.edu
}

Received for publication November 17, 1998, and accepted in revised form March 30, 1999.

\begin{abstract}
Conventional intracellular microelectrodes and injection of biocytin were used to study the actions of IL-1 $\beta$ and IL- 6 on electrical and synaptic behavior in morphologically identified guinea pig small intestinal submucous neurons. Exposure to nanomolar concentrations of either IL-1 $\beta$ or IL-6 stimulated neuronal excitability. The excitatory action consisted of depolarization of the membrane potential, decreased membrane conductance, and increased discharge of action potentials. Excitatory action of IL-1 $\beta$ was suppressed by the natural IL-1 $\beta$ human receptor antagonist. Electrical stimulation of sympathetic postganglionic axons evoked inhibitory postsynaptic potentials (IPSPs), and stimulation of cholinergic axons evoked nicotinic fast excitatory postsynaptic potentials (EPSPs). Both kinds of synaptic potentials occurred in neurons with uniaxonal morphology believed to be secretomotor neurons. Either IL- $1 \beta$ or IL- 6 suppressed the noradrenergic IPSPs and the fast EPSPs, and the two acted synergistically when applied in combination. Suppression of the IPSP resulted from presynaptic inhibition of the release of norepinephrine from sympathetic nerves. The results suggest that the presence of either or both inflammatory cytokines will release the sympathetic brake from secretomotor neurons to the intestinal crypts and from nicotinic synapses in the integrative microcircuits, where norepinephrine is known to have a presynaptic inhibitory action. This, in concert with excitation of secretomotor neurons, may lead to neurogenic secretory diarrhea.
\end{abstract}

J. Clin. Invest. 103:1309-1316 (1999).

\section{Introduction}

IL-1 $\beta$ and IL- 6 are proinflammatory cytokines that share several common biologic properties (1). They are expressed by a variety of cell types, but mainly they are made by monocytes and macrophages in response to a broad spectrum of stimuli including microbial derivatives, immune complexes, activated $\mathrm{T}$ cells, integrated actions of other cytokines, and psychogenic or environmental stress. Both cytokines are elevated in animal models of intestinal inflammation (2-5) and in ulcerative colitis and Crohn's disease, in which their levels reflect severity of disease activity (6-9). In patients with Crohn's disease, IL- $1 \beta$ and IL- 6 are not only elevated in regions of active disease but also to a lesser extent in bowel, where the mucosa appears endoscopically normal and from which biopsies are graded as histologically normal (9).

The cytokines are known to act at the neuronal level in both the peripheral and central nervous systems. When injected into the cerebral ventricles or specific brain nuclei, IL-1 $\beta$ has multiple actions that include thermogenesis, suppression of food intake, induction of slow-wave sleep, inhibition of $\mathrm{Ca}^{2+}$ channels in hippocampal neurons, and inhibition of long-term potentiation in the hypothalamus (10). During systemic inflammation induced by peripheral injection of Escherichia coli LPS, IL-1 $\beta$ gene expression becomes elevated in the central nervous system of rodents (11). In sheep, elevation of cerebral IL-1 $\beta$ accounts for the gas- trointestinal motor disturbances associated with LPS exposure (12). Application of IL- $1 \beta$ to spinal cord slices in vitro stimulates release of neuronal substance $P$, and this may be related to hyperalgesia in the animal (13). This effect in the spinal cord is reminiscent of increases in neuronal substance P evoked by IL-1 $\beta$ in the myenteric plexus of intestinal preparations (14).

In the peripheral nervous system, IL-1 $\beta$ acts on vagal afferent nerve terminals. Increased firing in vagal afferents is evoked by injection of IL- $1 \beta$ into the hepatic portal vein of rats (15). Vagal action is also suggested by elevated expression of c-Fos in the nucleus tractus solitarius, which is the brain stem projection site for vagal afferents activated by IL-1 $\beta$ (16). Pretreatment with IL- $1 \beta$ increases the sensitivity of gastric vagal afferents to fire in response to cholecystokinin, an effect that may underlie known anoretic actions of the cytokine (17). Finally, subdiaphragmatic vagotomy suppresses hyperthermic responses induced by IL- $1 \beta$ in the rat (18).

In the digestive tract, IL-1 $\beta$ suppresses basal and stimulus-evoked release of norepinephrine from sympathetic postganglionic nerve fibers and the release of acetylcholine from enteric neurons in rat longitudinal muscle/myenteric plexus preparations (19-21). IL-6 also suppresses norepinephrine release from sympathetic fibers in longitudinal muscle/myenteric plexus preparations and acts synergistically with IL- $1 \beta$ when the 2 cytokines are applied together (22). Enteric nerves appear to be 
a
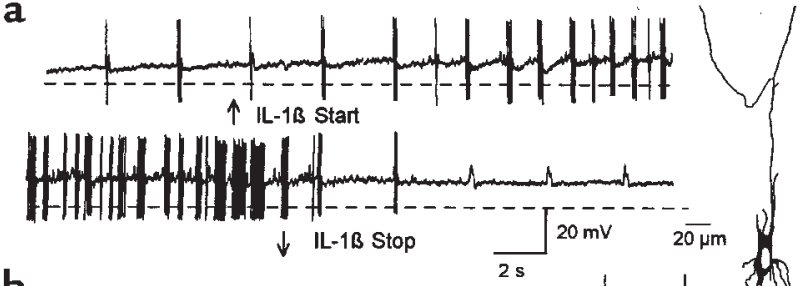

b

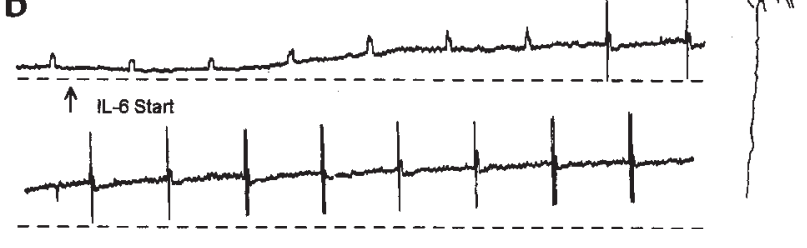

Figure 1

Application of IL-1b or IL- 6 depolarized and enhanced the excitability of a neuron in the submucous plexus. (a) IL-1 $13(10 \mathrm{nM})$ produced slowly activating depolarization of the membrane potential and enhanced excitability. Enhanced excitability is reflected by increased frequency of spike discharge during intraneuronal injection of constant-current depolarizing pulses and spontaneous spike discharge. The 2 traces are continuous. (b) IL-6 (10 nM) also produced slowly activating depolarization of the membrane potential and enhanced excitability in the same neuron. The 2 traces are continuous. The neuron had multiple long processes like Dogiel type II enteric neurons.

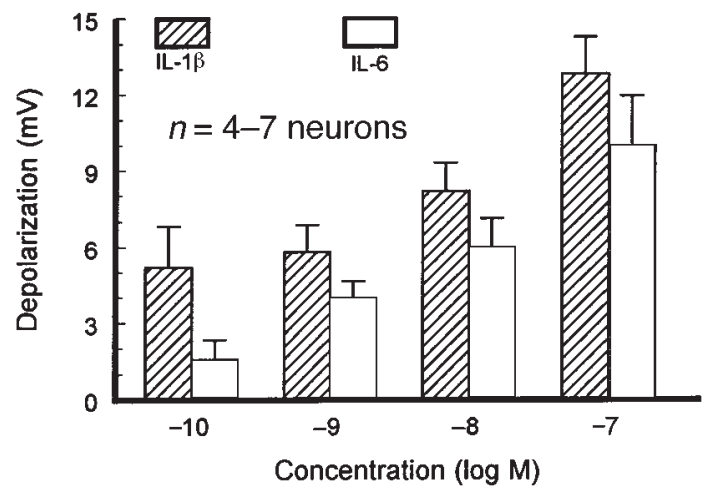

Figure 2

Amplitudes of the depolarizing responses to IL-1 $\beta$ and IL- 6 were concentration dependent.

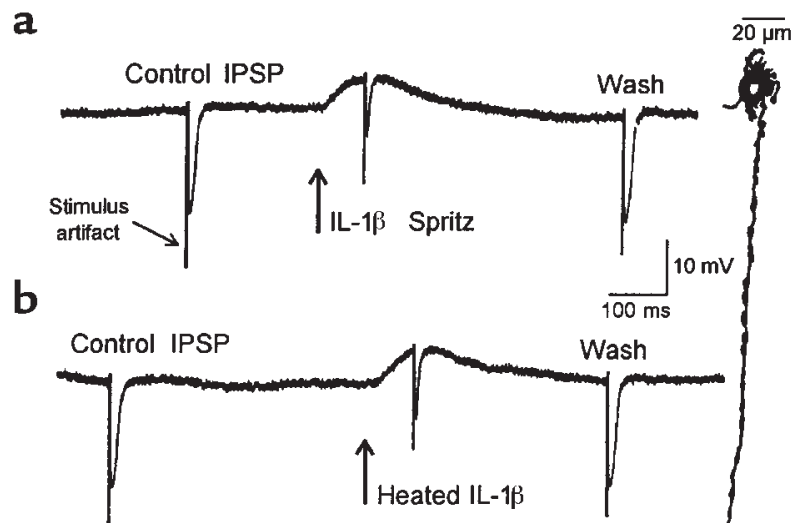

Figure 3

Depolarizing responses to IL-1 $\beta$ and suppression of IPSPs were unaffected by heating solutions containing IL-1 $\beta$. (a) Depolarization and suppression of the stimulus-evoked IPSP by unheated IL-1 $\beta$. (b) Depolarization and suppression of the IPSP by heated IL-1 $\beta$. The neuron had multiple short, dendritic-like processes and a single long process. involved in IL-1 $\beta$ suppression of responses to application of acetylcholine in small and large intestinal longitudinal muscle/myenteric plexus preparations because effects of the cytokine are blocked by tetrodotoxin (23).

The present study was undertaken in view of the importance of the cytokines in intestinal inflammation and the evidence that they have neuronal actions in both the central and autonomic nervous systems, including the enteric nervous system. We investigated the actions of IL- $1 \beta$ and IL- 6 on enteric neurons directly by recording their actions electrophysiologically with microelectrode impalements in morphologically identified enteric neurons. Preliminary results of the study have been published in abstract form (24).

\section{Methods}

Male Hartley-strain guinea pigs weighing 400-600 g were sacrificed by stunning and subsequent exsanguination, according to procedures approved by the Ohio State University Laboratory Animal Care and Use Committee. Segments of small intestine were removed $20 \mathrm{~cm}$ orad to the ileocecal junction. Preparations of the submucous plexus for electrophysiologic recording were microdissected as described previously (25). The preparations were mounted in a $2.0-\mathrm{mL}$ recording chamber that was perfused at a rate of $10-15 \mathrm{~mL} / \mathrm{min}$ with Krebs solution warmed to $37^{\circ} \mathrm{C}$ and gassed with $95 \% \mathrm{O}_{2} / 5 \% \mathrm{CO}_{2}$ to buffer at $\mathrm{pH}$ 7.3-7.4. The composition of the Krebs solution was (in $\mathrm{mM}) \mathrm{NaCl}, 120.9 ; \mathrm{KCl}, 5.9 ; \mathrm{MgCl}, 1.2 ; \mathrm{NaH}_{2} \mathrm{PO}_{4}, 1.2 ; \mathrm{NaHCO}_{3}$, 14.4; $\mathrm{CaCl}, 2.5$; and glucose, 11.5 .

Methods of intracellular recording from the submucous plexus are described in detail elsewhere (25). Transmembrane electrical potentials were recorded with conventional "sharp" microelectrodes. The microelectrodes were filled with $4 \%$ biocytin in $2 \mathrm{M} \mathrm{KCl}$ containing $0.05 \mathrm{M}$ Tris buffer ( $\mathrm{pH}$ 7.4). Resistances of the electrodes were 80-190 M $\Omega$. The marker dye (biocytin) was injected by the passage of hyperpolarizing current. The preamplifier (M767; World Precision Instruments, Sarasota, Florida, USA) had bridge circuitry for injection of electrical current pulses. Current pulses most commonly applied were 200 milliseconds in duration. All data were recorded on videotape for later analysis. Synaptic potentials were evoked by electrical shocks $(0.1-20 \mathrm{~Hz})$ applied focally to interganglionic connectives with Teflon-insulated platinum wire electrodes $20 \mu \mathrm{m}$ in diameter connected through stimulus-isolation units (Grass SIN5) to Grass S48 stimulators (Grass Instrument Division, Astro-Med Inc., Warwick, Rhode Island, USA). Chart records were made on Astro-Med thermal recorders. The amplitudes of the spikes shown in some of the figures here were blunted by the low-frequency response of the recorder.

At the end of each recording session, the anal end of the preparation was marked and fixative was added directly to the disposable recording chamber left overnight at $4^{\circ} \mathrm{C}$. The fixative contained $2 \%$ formaldehyde plus $15 \%$ of a saturated solution of picric acid. The preparations were cleared in 3 changes of dimethyl sulfoxide and three 10-minute washes with PBS. The preparations were reacted with avidin coupled to horseradish peroxidase, carried through a diaminobenzidine colordeveloping reaction, and then dehydrated in alcohol (26). The preparations were mounted in Canada balsam and examined with a microscope equipped with a Camera Lucida drawing tube (Nikon, Tokyo, Japan). All biocytin-injected neurons were identified as having a single long process (uniaxonal) or multiple long processes (Dogiel type II) (27). Generally accepted criteria for electrophysiologic and morphologic classification of AH- and S-type neurons were used $(28,29)$.

Actions of IL- $1 \beta$ and IL- 6 were studied by pressure microejection or by application in the superfusion solution. 


\section{a}

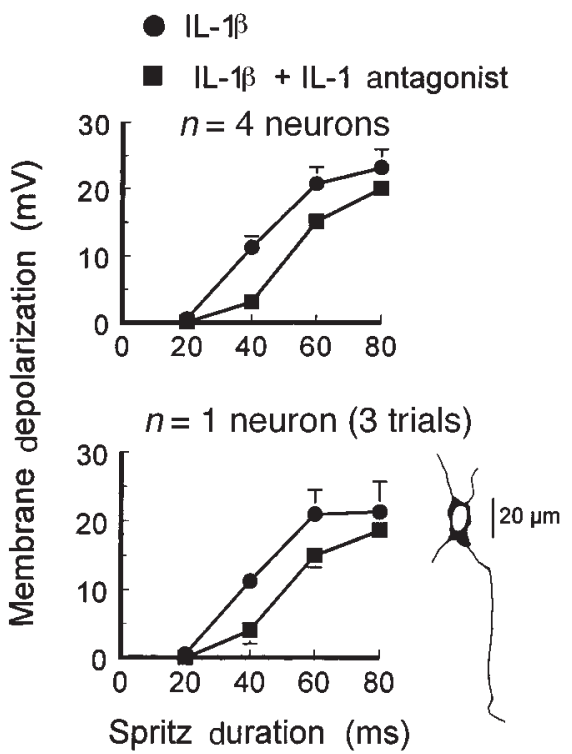

Figure 4

Recombinant IL-1 natural antagonist suppressed depolarizing responses to IL-1 $\beta$. (a) Progressively longer pressure ejection pulses of $0.1 \mu \mathrm{M} \mathrm{IL-1} \beta$ (i.e., 20-80 milliseconds) evoked duration-dependent depolarization in 4 neurons, of which 3 had uniaxonal morphology and 1 had multiple long processes like Dogiel type II neurons. Pretreatment with 3 nM IL-1 antagonist resulted in a rightward shift of the duration-response curve. (b) Same kind of data as in a for 3 trials in a single neuron with Dogiel type II-like morphology.

Micropipettes (10 $\mu \mathrm{m}$ in diameter) manipulated with the tip close to the impaled neurons were used to microeject the substances. Pressure pulses of nitrogen with predetermined force and duration were applied to the micropipettes through electronically controlled solenoid valves.

Chemical agents used and sources were as follows. Norepinephrine, tetrodotoxin, phentolamine, human recombinant IL6, biocytin, and pirenzepine were from Sigma Chemical Co. (St. Louis, Missouri, USA). Human recombinant IL-1 $\beta$ and IL-1 natural receptor antagonist were obtained from Calbiochem-Novabiochem Corp. (La Jolla, California, USA) and Bachem California Inc. (Torrance, California, USA), respectively. Peninsula Laboratories (Belmont, California, USA) was the source of [Trp7, B-Ala8]-neurokinin $A_{4-10}$. Renzapride (BRL 24924) was a gift from SmithKline Beecham (Betchworth, United Kingdom). Data are expressed as means \pm SE. Student's $t$ test for paired or unpaired values was used to evaluate significance of differences for means of resting membrane potential and neuronal input resistance.

\section{Results}

Excitatory actions of IL-1 $\beta$ and IL-6. IL- $1 \beta$ was studied in 23 AH-type and 39 S-type neurons, and IL- 6 was studied in $23 \mathrm{AH}$-type and $41 \mathrm{~S}$-type neurons in the submucous plexus. All neurons had resting membrane potentials greater than $-45 \mathrm{mV}$. Classification characteristics of $\mathrm{AH}$ - and S-type enteric neurons were reviewed by Wood (28) and Bornstein et al. (29). Application of IL-1 $\beta$ in the bathing medium $(0.1-100 \mathrm{nM})$ or by pressure microejection $(0.1 \mu \mathrm{M})$ stimulated neuronal excitability in 17 of $23 \mathrm{AH}$ neurons and 28 of $39 \mathrm{~S}$ neurons. IL- 6 in similar concentrations enhanced excitability in 16 of $23 \mathrm{AH}$ and 21 of $41 \mathrm{~S}$ neurons. The excitatory action for both cytokines consisted of depolarization of the membrane potential coincident with increased input resistance. IL$1 \beta(100 \mathrm{nM})$ increased the input resistance by $28.3 \pm$ $10.5 \%$ in 13 neurons, and 100 nM IL-6 increased the input resistance by $19.2 \pm 5.8 \%$ in 7 neurons.

Enhanced excitability was apparent as spontaneous spike discharge or repetitive discharge during intraneuronal injection of depolarizing current pulses (Figure 1). Suppression of the characteristic hyperpolarizing afterpotentials in $\mathrm{AH}$ neurons accompanied the changes in excitability. Amplitudes of the depolarizing responses to IL-1 $\beta$ and IL- 6 increased in direct relation to the concentration of the cytokine or to the duration of pressure ejection pulses (Figure 2). All effects were reversed by washout from the recording chamber. Heating the solution containing IL- $1 \beta$ to boiling did not alter the depolarizing action of the cytokine (Figure 3 ).

The depolarization evoked by 80 -millisecond micropressure pulses of $0.1 \mu \mathrm{M}$ IL- $1 \beta$ was not reduced by the presence of the serotonergic (5-HT1P) antagonist BRL $24924(10 \mu \mathrm{M})$ in the bathing solution. Mean depolarization to IL- $1 \beta$ was $24.25 \pm 5.0 \mathrm{mV}$ for 5 neurons in the absence of BRL 24924 and $24.54 \pm 5.4 \mathrm{mV}$ for the same neurons in the presence of BRL $24924(P>0.05)$. The depolarization evoked by 40 -millisecond micropressure pulses of $0.1 \mu \mathrm{M}$ IL-1 $\beta$ was also not reduced by the presence of $1 \mu \mathrm{M}$ of the neurokinin-3 receptor antagonist [Trp7, B-Ala8]-neurokinin $\mathrm{A}_{4-10}$ in the bathing solution. Mean depolarization to IL-1 $\beta$ was $19.6 \pm 4.6 \mathrm{mV}$ for 7 neurons in the absence of the antagonist and $20.1 \pm 4.0$ $\mathrm{mV}$ for the same neurons in the presence of antagonist $(P$ $>0.05)$. The depolarizing responses evoked by 40 -millisecond micropressure pulses of $0.1 \mu \mathrm{M}$ IL-1 $\beta$ were also

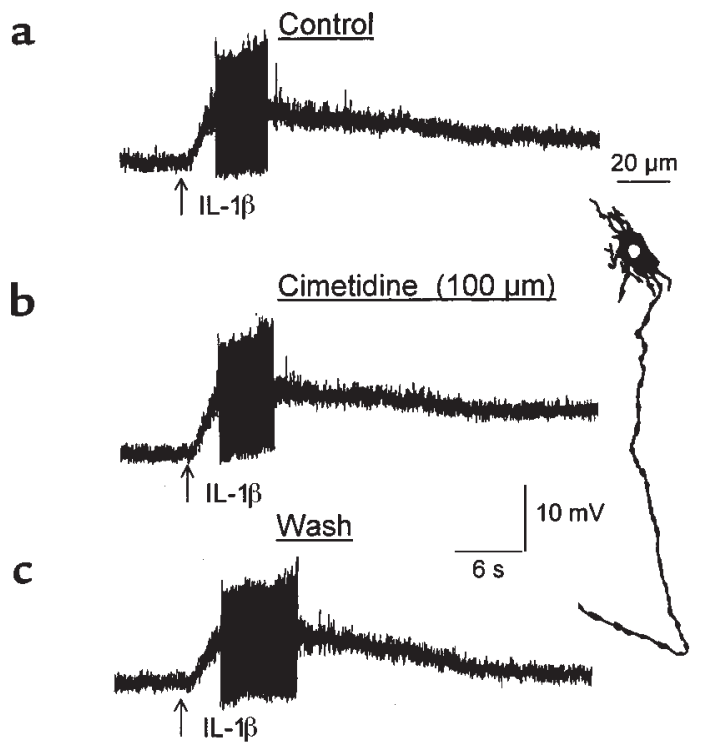

Figure 5

Application of cimetidine did not suppress the depolarizing action and enhanced excitability produced by IL-1 $\beta$ in a neuron with multiple short, dendritic-like processes and a single long process. (a) Depolarization and action potential discharge evoked by a microejection pulse of IL-1 $\beta$. (b) Responses to IL-1 $\beta$ were unchanged in the presence of cimetidine. (c) Responses to IL-1 $\beta$ after washout of cimetidine. Failure of cimetidine to suppress the response to IL-1 $\beta$ suggested that the action was directly on the neuron rather than due to release of histamine from mast cells. 
a

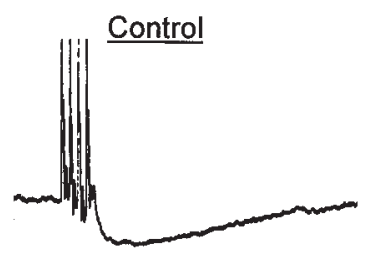

b

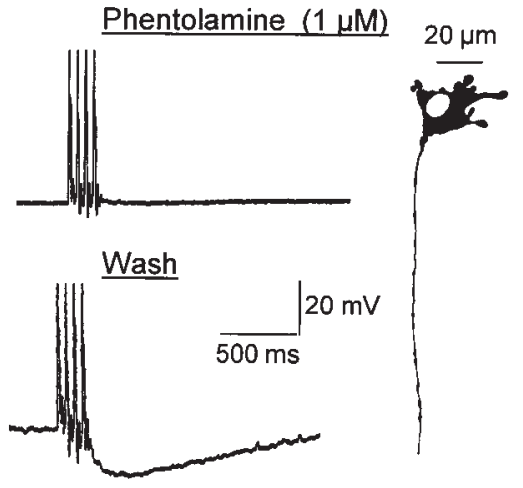

not reduced by the presence of $100 \mu \mathrm{M}$ of the $\mathrm{M}_{1}$ muscarinic receptor antagonist pirenzepine in the bathing solution. Mean depolarization to IL-1 $\beta$ was $19.5 \pm 4.5 \mathrm{mV}$ for 6 neurons in the absence of the antagonist and $19.8 \pm$ $4.4 \mathrm{mV}$ for the same neurons in the presence of antagonist $(P>0.05)$. These results suggested that the slowly activating depolarization evoked by IL- $1 \beta$ was direct rather than secondary to release of serotonin, substance $\mathrm{P}$, or acetylcholine from nerve terminals or paracrine signaling cells (e.g., mast cells).

Excitatory (depolarizing) responses to pressure ejection pulses of IL- $1 \beta$ were suppressed by the natural IL- 1 receptor antagonist when the antagonist was applied in the bathing solution at concentrations greater than 1 nM (Figure 4). Quasi concentration-response curves obtained by progressively increasing the duration of pressure ejection pulses showed a parallel rightward shift suggestive of competitive interactions between the agonist and antagonist at the IL- $1 \beta$ receptor (Figure 4 ).

The excitatory responses to the cytokines were reminiscent of excitation of submucous neurons produced by degranulation of antigen-sensitized mucosal mast cells $(30,31)$. One of the mediators released by mast cell degranulation is histamine, which acts at the histamine $\mathrm{H}_{2}$ receptor subtype to depolarize and enhance excitability of neurons in the guinea pig submucous plexus (30-32).

The $\mathrm{H}_{2}$ histamine receptor antagonist cimetidine was used to determine whether the excitatory responses to IL- $1 \beta$ or IL- 6 might be a secondary effect due to degranulation of mast cells and the release of histamine. Cimetidine is known to block the excitatory actions of exogenously applied histamine and histamine released by mast cells during antigen exposure in sensitized guinea pig bowel in vitro (30-32).

Pretreatment with cimetidine blocked the neuronal excitatory responses to micropressure pulses of histamine, but it did not significantly change the depolarizing responses to either cytokine (Figure 5). This suggested that their excitatory actions were directly on the neurons and not secondary to mast cell activation by the cytokine.

Excitatory responses evoked by either IL- $1 \beta$ or IL- 6

\section{Figure 6}

Focal electrical stimulation of sympathetic noradrenergic synaptic inputs to submucous plexus neurons evoked IPSPs. Application of the $\alpha$-noradrenergic receptor antagonist phentolamine reversibly abolished the stimulus-evoked IPSPs. (a) Control IPSP. (b) Blockade of the IPSP in the presence of $1 \mu \mathrm{M}$ phentolamine. (c) Recovery of IPSP after washout of phentolamine The neuron had multiple short, clublike dendrites and a single long process. (membrane depolarization) were unaffected by pretreatment with $300 \mathrm{nM}$ tetrodotoxin or solutions with depleted $\mathrm{Ca}^{2+}$ and elevated $\mathrm{Mg}^{2}$. In Krebs solution with $300 \mathrm{nM}$ tetrodotoxin, $1.25 \mathrm{mM} \mathrm{Ca}^{2+}$, and $16 \mathrm{mM} \mathrm{Mg}^{2+}$, the mean depolarization evoked by 40 -millisecond micropressure pulses of $100 \mathrm{nM} \mathrm{IL}-1 \beta$ was $16.7 \pm 2.8 \mathrm{mV}$ for 7 neurons, compared with $16.3 \pm 3.5 \mathrm{mV}(P>0.05)$ for the same neurons in normal Krebs solution. This suggested direct action of the cytokines because tetrodotoxin was expected to block axonal conduction in the plexus and thereby prevent input to the recorded neuron from synaptically connected neurons elsewhere in the microcircuit.

Suppression of inbibitory postsynaptic potentials by $I L-1 \beta$ and

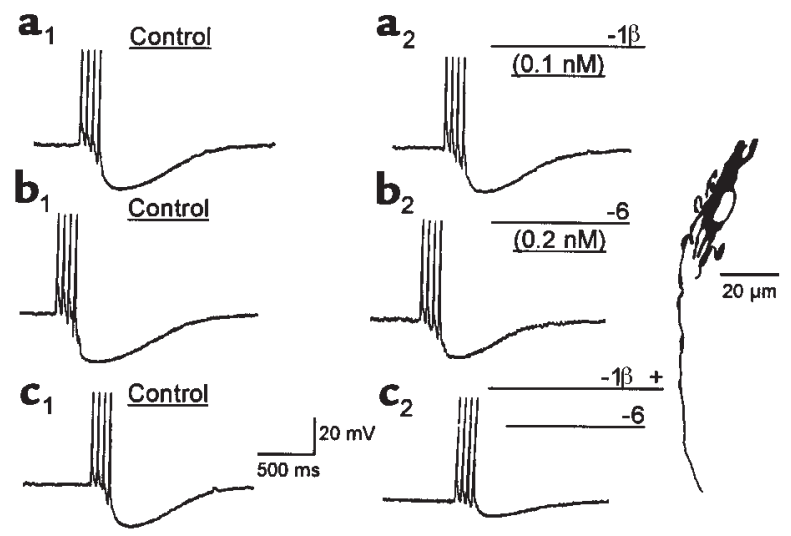

Figure 7

Focal electrical stimulation of sympathetic noradrenergic synaptic inputs to submucous plexus neurons evoked IPSPs. Application of IL-1 $\beta$ or IL-6 in the superfusion solution suppressed the stimulus-evoked IPSPs. Simultaneous application of the 2 cytokines resulted in a greater suppression of the IPSPs than the sum of the two when each was added alone. (a) IPSPs evoked by electrical stimulation before application of IL-1 $\beta$ (control) and in the presence of $0.1 \mathrm{nM} \mathrm{IL-1 \beta}$. (b) IPSPs evoked by electrical stimulation before application of IL-6 (control) and in the presence of 0.2 nM IL-6. (c) Simultaneous application of 0.1 nM IL-1 $\beta$ and 0.2 nM IL- 6 produced greater suppression than the sum of individual exposures to IL$1 \beta$ and IL-6 (see Figure 8). The neuron had multiple short, clublike dendrites and a single long process. 


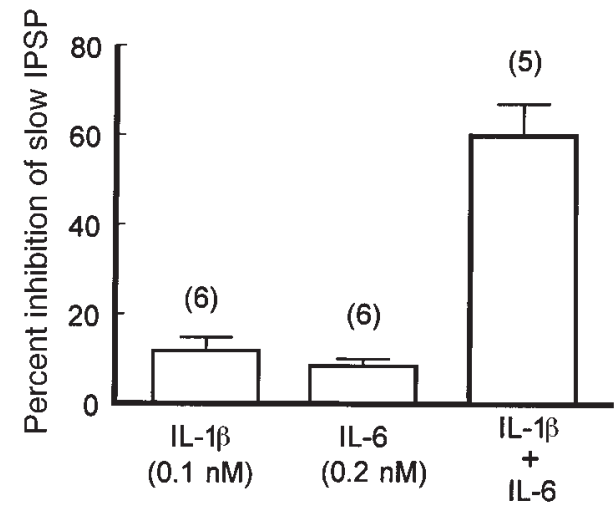

Figure 8

IL-1 $\beta$ and IL-6 each suppressed stimulus-evoked IPSPs in submucous neurons and acted synergistically when applied in combination in the superfusion solution. Parenthetical number above each bar represents number of neurons.

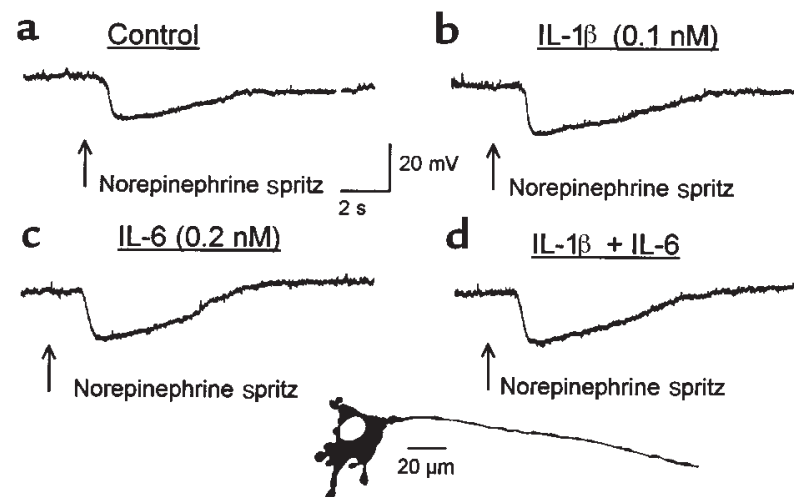

Figure 9

Neither IL-1 $\beta$ nor IL- 6 suppressed hyperpolarizing responses to norepinephrine in submucous plexus neurons. (a) Control response to micropressure pulse ("spritz") of $10 \mu \mathrm{M}$ norepinephrine. (b) IL-1 $\beta$ did not suppress the response to norepinephrine in the same neuron. (c) Control response to spritz of $10 \mu \mathrm{M}$ norepinephrine. (d) IL- 6 did not suppress the response to norepinephrine in the same neuron. The neuron had multiple short, clublike dendrites and a single long process.

$I L-6$. Focal electrical stimulation of sympathetic noradrenergic synaptic inputs to the submucous neurons evoked inhibitory postsynaptic potentials (IPSPs) (Figure 6). IPSPs were found in both AH neurons with Dogiel type II morphology and S neurons with Dogiel type I morphology. Mimicry of the IPSPs by micropressure application of norepinephrine and blockade of the IPSPs and the responses to norepinephrine by $1 \mu \mathrm{M}$ of the $\alpha$ adrenoceptor antagonist phentolamine in 7 neurons (Figure 6) were consistent with earlier reports that the noradrenergic IPSPs in guinea pig submucous plexus neurons are mediated by $\alpha$-noradrenergic receptors $(25$, $33)$. The mean amplitude of the slow IPSPs was $20.5 \pm 1.1$ $\mathrm{mV}$ (range: 6-30 mV) for 30 neurons. Application of IL$1 \beta$ or IL- 6 in the superfusion solution reversibly suppressed or abolished the stimulus-evoked IPSPs (Figure 7). Application of $0.1 \mathrm{nM}$ IL- $1 \beta$ or $0.2 \mathrm{nM}$ IL- 6 suppressed the IPSPs by $10-20 \%$ IL- $1 \beta$ and IL- 6 applied together in the same concentrations acted synergistically to suppress the IPSPs by a mean of $60 \pm 8.2 \%$ (Figure 8 ).

Micropressure application of norepinephrine evoked hyperpolarizing responses in the submucous neurons (Figure 9). Like the stimulus-evoked IPSPs, the hyperpolarizing responses to norepinephrine were suppressed by the $\alpha$ noradrenergic antagonist phentolamine. IL-1 $\beta$, IL-6,or the 2 cytokines in combination the hyperpolarizing responses evoked by norepinephrine (Figure 9). In the presence of 0.1 $\mathrm{nM}$ IL-1 $\beta$ or $0.2 \mathrm{nM}$ IL-6, the mean hyperpolarizing responses to norepinephrine were $25.7 \pm 2.4 \mathrm{mV}$ and 27.5 $\pm 3.6 \mathrm{mV}$, respectively, for 15 neurons. These were not significantly different $(P>0.05)$ from the control value of 25.1 $\pm 3.0 \mathrm{mV}$. This suggested that the site of action of the cytokines was at presynaptic inhibitory receptors on the noradrenergic nerve terminals and that suppression of the IPSPs resulted from inhibition of norepinephrine release from the terminals.

Suppression of fast excitatory postsynaptic potentials by $I L-1 \beta$ and IL-6. Focal electrical stimulation applied to interganglionic connectives in the submucous plexus evoked fast excitatory postsynaptic potentials (EPSPs) characteristic of the well-documented nicotinic EPSPs found to occur in enteric neurons (34). Fast EPSPs were found primarily in S-type neurons with uniaxonal morphology. Mean amplitude of the fast EPSPs was $6.4 \pm 1.4 \mathrm{mV}$ for 25 neurons. Pressure microejection (40-millisecond pulses) of $0.1 \mu \mathrm{M}$ IL- $1 \beta$ or $0.2 \mu \mathrm{M}$ IL- 6 reversibly suppressed or abolished the stimulus-evoked EPSPs (Figure $10)$. Micropressure application of $0.1 \mathrm{nM}$ IL- $1 \beta$ or 0.2 nM IL-6 suppressed the EPSPs in direct relation to the duration of the constant-pressure microejection pulses (Figure 10). IL-1 $\beta$ and IL-6 applied together in the same concentrations acted synergistically to suppress the EPSPs (Figure 11).

Micropressure application of acetylcholine evoked depolarizing responses that mimicked the fast EPSPs (Figure 12). IL-1 $\beta$, IL- 6 , or the 2 cytokines in combination did not alter the depolarizing responses evoked by acetylcholine

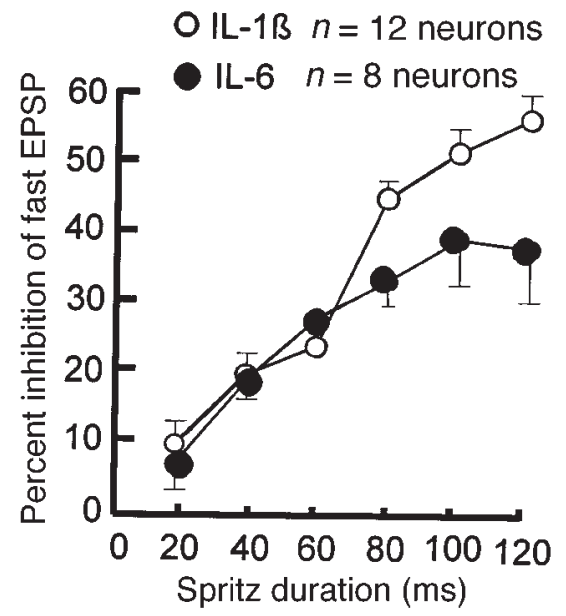

Figure 10

Focal electrical stimulation of nicotinic synaptic inputs to submucous plexus neurons evoked EPSPs. Application of IL-1 $\beta$ or IL-6 suppressed the EPSPs. Suppression of EPSPs by either IL-1 $\beta$ or IL- 6 was directly related to the duration of constant-pressure microejection pulses with $0.1 \mu \mathrm{M}$ in the ejection pipette. 
a
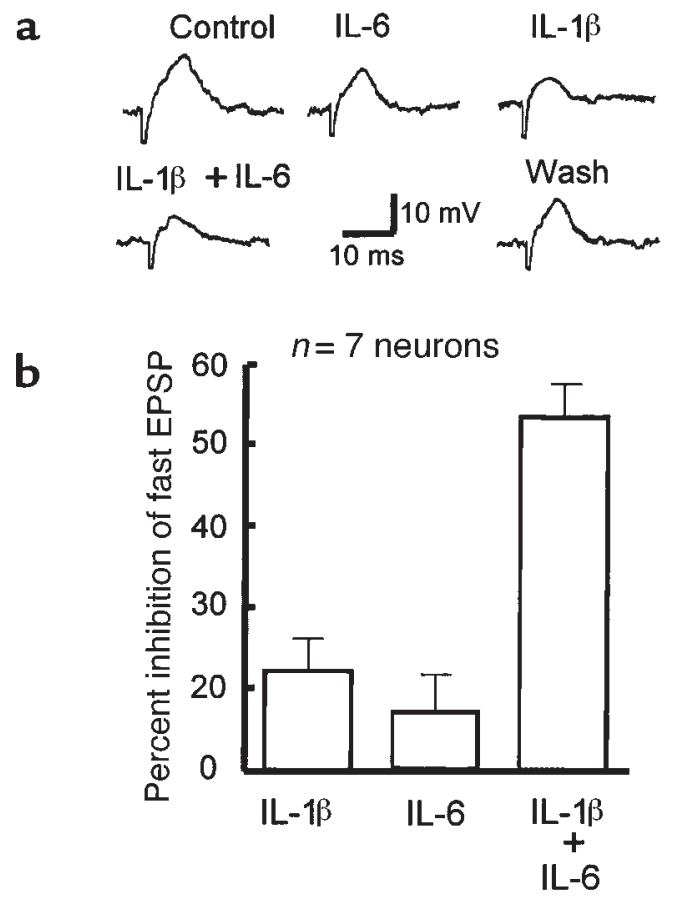

Figure 11

Focal electrical stimulation of nicotinic synaptic inputs to submucous plexus neurons evoked EPSPs. Application of IL- $1 \beta$ or IL- 6 suppressed the EPSPs. Simultaneous application of the 2 cytokines resulted in a greater suppression than the sum of the two added separately. (a) EPSP evoked by electrical stimulation before application of the cytokines (control) followed by $0.2 \mathrm{nM} \mathrm{IL-}-6,0.1 \mathrm{nM} \mathrm{IL-} 1 \beta$, a combination of $0.1 \mathrm{nM} \mathrm{IL-} 1 \beta$ and $0.2 \mathrm{nM}$ IL-6, and washout. (b) Simultaneous application of IL-1 $\beta$ and IL-6produced greater suppression than the sum of individual exposures to IL-1 $\beta$ and IL-6.

(Figure 12). The mean amplitude of depolarizing responses evoked by micropressure application of $100 \mu \mathrm{M}$ acetylcholine was $27.7 \pm 6.4 \mathrm{mV}$ for 6 neurons. In the presence of $0.1 \mathrm{nM}$ IL- $1 \beta$ or $0.2 \mathrm{nM}$ IL- 6 , the amplitude of the acetylcholine-evoked responses was $28.3 \pm 6.7 \mathrm{mV}$ or 27.4 $\mathrm{mV}$, respectively, for the same 6 neurons. This suggested that the site of action of the cytokines was at presynaptic inhibitory receptors on the cholinergic nerve terminals and that suppression of the EPSPs resulted from inhibition of acetylcholine release from the terminals.

\section{Discussion}

The results suggest that the cytokines IL- $1 \beta$ and IL- 6 have direct actions on neurons in the submucous plexus of guinea pig small intestine. Three kinds of actions were found. One was an excitatory action at the level of the membranes of the neuronal cell bodies; the second was suppression of slow IPSPs; and the third was suppression of nicotinic fast EPSPs.

The excitatory action mimicked slow synaptic excitatory responses characteristically found in enteric neurons (reviewed in ref. 34). Excitation evoked by the cytokines also mimicked the slow excitatory effects produced by paracrine release of histamine from mucosal mast cells (30-32). Failure of a histamine receptor antagonist and blockade of axonal conduction by tetrodotoxin to suppress the excitatory responses to the cytokines is evidence for direct action at the somal membranes of the neurons. The excitatory action could not be attributed to secondary effects of mast cell degranulation.

A second action of IL-1 $\beta$ and IL- 6 was suppression of IPSPs evoked by stimulation of release of norepinephrine from sympathetic postganglionic nerve fibers. This appeared to be a direct action at presynaptic inhibitory receptors on the noradrenergic terminals. Suppression of the noradrenergic IPSP may be the electrophysiologic correlate of suppression of $\left[{ }^{3} \mathrm{H}\right]$ norepinephrine release that has been reported to be an action of IL- $1 \beta$ in studies of the small bowel (20). Our findings that IL- $1 \beta$ and IL- 6 act synergistically in suppressing the noradrenergic IPSPs in the guinea pig are consistent with results showing synergism between the 2 cytokines in suppressing release of $\left[{ }^{3} \mathrm{H}\right]$ norepinephrine from longitudinal muscle/myenteric plexus preparations of rat small intestine (22).

A third action of IL-1 $\beta$ and IL- 6 was suppression of EPSPs evoked by stimulation of acetylcholine release at fast nicotinic synapses. This appeared to be a direct action at presynaptic inhibitory receptors on the cholinergic nerve terminals. Suppression of the cholinergic EPSP may be the electrophysiologic correlate of suppression of $\left[{ }^{3} \mathrm{H}\right]$ choline release from within the enteric nervous system that has been reported as another action of IL-1 $\beta$ (21).

Presynaptic inhibition by IL- $1 \beta$ may be a generalized phenomenon in the nervous system. In ganglia of the pelvic plexus in guinea pigs, it acts to suppress nicotinic EPSPs in much the same way as we found for enteric ganglia (35). In brain slice preparations from the basolateral amygdala of rats, IL- $1 \beta$ acts at presynaptic inhibitory receptors to suppress fast excitatory and fast and slow inhibitory postsynaptic potentials evoked by electrical stimulation in either the stria terminalis or the lateral amygdala (36).

An aspect of the pathophysiologic significance of the neuronal actions of the 2 cytokines is illustrated by the model in Figure 13. Secretomotor neurons to the intes-

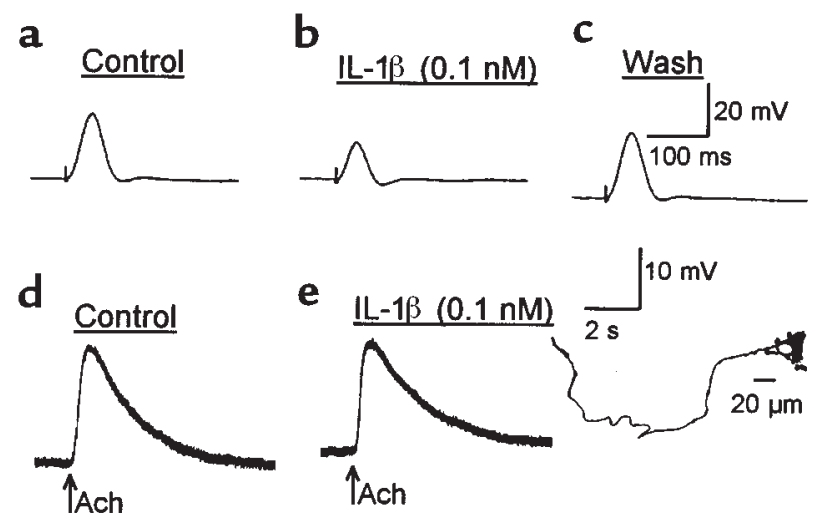

Figure 12

IL-1 $\beta$ reversibly suppresses stimulus-evoked fast EPSPs, but not responses to acetylcholine, in submucous neurons. (a) Control. (b) Suppression of EPSP in presence of $0.1 \mathrm{nM} \mathrm{IL-1 \beta}$. (c) Recovery of IPSP after washout of IL-1 $\beta$. (d) Control response to micropressure pulse of $100 \mu \mathrm{M}$ acetylcholine. (e) Responses to same micropressure pulse of acetylcholine in presence of $0.1 \mathrm{nM} \mathrm{IL}-1 \beta$ were unchanged. Morphology of the neuron was uniaxonal with multiple short, clublike dendrites. 


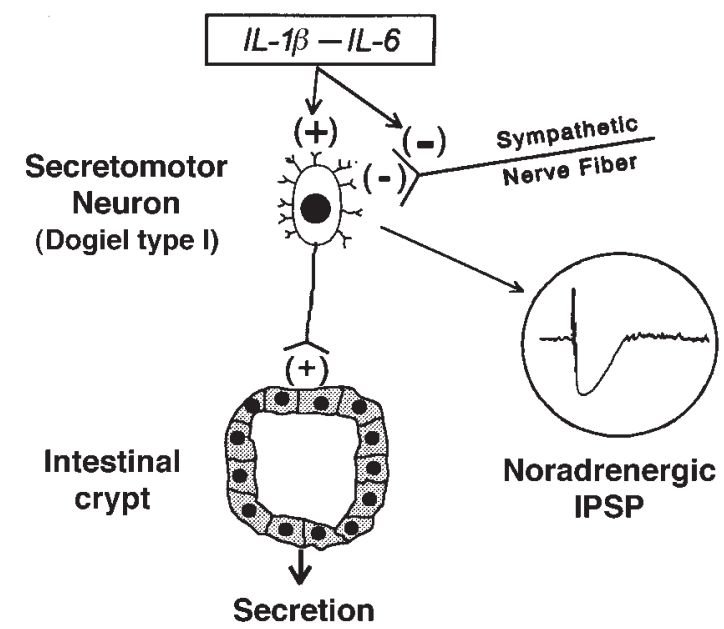

tinal crypts are located in the submucous plexus. When secretomotor neurons fire, they release vasoactive intestinal polypeptide or acetylcholine at the neuroepithelial junctions, and this stimulates the secretion of water and electrolytes into the crypt lumen (37). Hyperactivity of the secretomotor neurons is associated with a state of secretory diarrhea. Two actions of the cytokines are expected to contribute to production of the secretory state. One is direct excitation of the secretomotor neurons and/or interneurons that provide excitatory synaptic drive to the secretomotor neurons. Second is the inhibitory action on noradrenergic transmission to the secretomotor neurons and/or synaptically connected interneurons. This effectively nullifies any sympathetic braking action on the secretomotor neurons to permit maximal secretomotor firing rates and hyperstimulation of mucosal secretion.

Apart from submucous secretomotor neurons, a major component of sympathetic input to the intestine acts at presynaptic terminals to prevent release of excitatory transmitter substances that mediate fast or slow transmission at synapses within the integrated circuits of the enteric nervous system (38). These axo-axonal synapses, which have been described ultrastructurally, function to inactivate the excitatory synaptic circuitry that mediates intestinal motility (39). Application of norepinephrine or electrical stimulation of sympathetic postganglionic fibers in the intestinal mesentery reduces or abolishes fast nicotinic transmission in the enteric networks $(40,41)$. This occurs without any change in the somal membrane properties during exposure to norepinephrine and without any change in the depolarizing responses of the neurons to microejected acetylcholine. Both findings are evidence that the mechanism of action of norepinephrine is blockade of release of acetylcholine from presynaptic nerve terminals. Based on the neurophysiology at the synaptic interface between the sympathetic and enteric divisions of the autonomic nervous system, the significance of cytokineinduced blockade of norepinephrine release at excitatory synapses is expected to be prevention of sympathetic inactivation of the enteric microcircuits that generate intestinal motor activity and other intestinal behaviors associated with the inflammatory state.

The significance of presynaptic suppression of fast

\section{Figure 13}

Conceptual model for actions of IL-1 $\beta$ and IL- 6 in the enteric nervous system. Both cytokines act directly to increase excitability of neurons and to suppress release of norepinephrine at sympathetic synapses on submucous neurons. Stimulation of submucosal secretomotor neurons is expected to evoke secretion from mucosal crypts and may account in part for diarrheal symptoms associated with proinflammatory cytokines. Inactivation of sympathetic braking action on secretomotor neurons facilitates secretion and may also contribute to diarrheal symptoms.

excitatory neurotransmission in the enteric nervous system is less clear. In a situation in which multiple neurons in a circuit have stimulated excitability (i.e., by the cytokine), it may be that presynaptic inhibition at multiple synaptic transfer points in the circuit provides a simultaneous braking action that prevents runaway excitation at the circuit level. Braking action of this nature could compensate for the loss of the sympathetic brake that results from the action of the cytokines to suppress norepinephrine release.

\section{Acknowledgments}

This study was supported by National Institutes of Health grant RO1 DDK-37238.

1. Dinarello, C.A. 1994. Interleukin-1. Adv. Pharmacol. 25:21-51.

2. Kahn, I., and Collins, S.M. 1994. Expression of cytokines in the longitudinal muscle myenteric plexus of the inflamed intestine of rat. Gastroenterology. 107:691-700.

3. Thomas, T.K., et al. 1991. Evaluation of an interleukin-1 receptor antagonist in the rat acetic acid-induced colitis model. Agents Actions. 34:187-190

4. Cominelli, G., et al. 1990. Interleukin (IL-1) gene expression, synthesis, and effect of specific IL-1 receptor blockade in rabbit immune complex colitis. J. Clin. Invest. 86:972-980.

5. Rachmilewitz, D., et al. 1989. Inflammatory mediators of experimental colitis in rats. Gastroenterology. 97:326-327.

6. Mahida, Y.R., Wu, K., and Jewell, D.P. 1989. Enhanced production of interleukin- $1 \beta$ by mononuclear cells isolated from mucosa with active ulcerative colitis and Crohn's disease. Gut. 30:835-838.

7. Ligumsky, M., Simon, P.L., Karmeli, F., and Racmilewitz, D. 1990. Role of IL-1 in inflammatory bowel disease. Enhanced production during active disease. Gut. 31:686-689.

8. Stevens, C., et al. 1992. Tumor necrosis factor-alpha, interleukin-1 beta and interleukin-6 expression in inflammatory bowel disease. Dig. Dis. Sci. 37:818-826.

9. Reimund, J.M., et al. 1996. Increased production of tumour necrosis factor-alpha, interleukin- 1 beta, and interleukin- 6 by morphologically normal intestinal biopsies from patients with Crohn's disease. Gut. 39:684-689.

10. Rothwell, N.J., and Luheshi, G. Pharmacology of interleukin-1 actions in the brain. Adv. Pharmacol. 25:1-20.

11. Wong, M.L., Bongiorno, P.B., Rettori, V., Mccann, S.M., and Licinio, J. 1997. Interleukin (IL) 1 beta, IL-1 receptor antagonist, IL-10, and IL-13 gene expression in the central nervous system and anterior pituitary during systemic inflammation: pathophysiological implications. Proc. Natl. Acad. Sci. USA. 94:227-232.

12. Plaza, M.A., Fioramonti, J., and Bueno, J. 1997. Role of central interleukin-1 $\beta$ in gastrointestinal motor disturbances induced by lipopolysaccharide in sheep. Dig. Dis. Sci. 42:242-250.

13. Malcangio, M., Bowery, N.G., Flower, R.J., and Perretti, M. 1996. Effect of interleukin-1 beta on the release of substance $\mathrm{P}$ from rat isolated spinal cord. Eur. J. Pharmacol. 299:113-118.

14. Hurst, S.M., Stanisz, A.M., Sharkey, K.A., and Collins, S.M. 1993. Interleukin- 1 beta induced increase in substance- $\mathrm{P}$ in rat myenteric plexus. 
Gastroenterology. 105:1754-1760.

15. Niijima, A. 1992. The afferent discharges from sensors for interleukin-1 beta in hepatoportal system in the anesthetized rat. J. Physiol. (Lond.) 446:236P.

16. Ericsson, A., Kovacs, K.J., and Sawchenko, P.E. 1994. A functional anatomical analysis of central pathways subserving the effects of interleukin-1 on stress-related neuroendocrine neurons. J. Neurosci. 14:897-913.

17. Bucinskaite, V., Kurosawa, M., Miyasaka, K., Funakoshi, A., and Lundeberg, T. 1997. Interleukin-1 beta sensitizes the response of the gastric vagal afferent to cholecystokinin in rat. Neurosci. Lett. 229:33-36.

18. Watkins, L.R., et al. 1995. Blockade of interleukin-1 induced hyperthermia by subdiaphragmatic vagotomy: evidence for vagal mediation of immune brain communication. Neurosci. Lett. 183:27-31.

19. Collins, S.M., et al. 1992. Effect of inflammation on enteric nerves: cytokine-induced changes in neurotransmitter content and release. Ann. NY Acad. Sci. 664:415-424.

20. Hurst, S., and Collins, S.M. 1993. Interleukin-1a modulation of norepinephrine release from rat myenteric nerves. Am. J. Physiol. 27:G30-G35.

21. Main, C., Blennerhassett, P., and Collins, S.M. 1993. Human recombinant interleukin-1-beta suppresses acetylcholine release from rat myenteric plexus. Gastroenterology. 104:1648-1654.

22. Ruhl, A., Hurst, S., and Collins, S.M. 1994. Synergism between interleukins- 1 beta and -6 on noradrenergic nerves in rat myenteric plexus. Gastroenterology. 107:993-1001.

23. Aube, A.C., et al. 1996. Inhibition of acetylcholine induced intestinal motility by interleukin-1 beta in the rat. Gut. 39:470-474

24. Xia, Y., Cooke, H.J., and Wood, J.D. 1996. Interleukin-1 beta and interleukin- 6 excite neurons and suppress inhibitory noradrenergic neurotransmission in the submucous plexus of guinea-pig small intestine. Gastroenterology. 110:A783. (Abstr.)

25. Zafirov, D.H., Cooke, H.J., and Wood, J.D. 1993. Elevation of cAMP facilitates noradrenergic transmission in submucous neurons of guinea-pig ileum. Am. J. Physiol. 264:G442-G446.

26. Bornstein, J.C., Furness, J.B., Smith, T.K., and Trussell, D.C. 1991. Synaptic responses evoked by mechanical stimulation of the mucosa in morphologically characterized myenteric neurons of the guinea-pig ileum. J. Neurosci. 11:505-518.

27. Furness, J.B., Bornstein, J.C., and Trussell, D.C. 1988. Shapes of nerve cells in the myenteric plexus of the guinea pig small intestine revealed by the intracellular injection of dye. Cell Tissue Res. 254:561-571.

28. Wood, J.D. 1994. Application of classification schemes to the enteric nervous system. J. Auton. Nerv. Syst. 48:17-29.

29. Bornstein, J.C., Furness, J.B., and Kunze, W.A.A. 1994. Electrophysiological characterization of myenteric neurons: how do classification schemes relate? J. Auton. Nerv. Syst. 48:1-15.

30. Frieling, T., Cooke, H.J., and Wood, J.D. 1994. Neuroimmune communication in the submucous plexus of guinea-pig colon after sensitization to milk antigen. Am. J. Physiol. 267:G1087-G1093.

31. Frieling, T., Palmer, J.M., Cooke, H.J., and Wood, J.D. 1994. Neuroimmune communication in the submucous plexus of guinea pig colon after infection with Trichinella spiralis. Gastroenterology. 107:1602-1609.

32. Frieling, T., Cooke, H.J., and Wood, J.D. 1992. Histamine receptors on submucous neurons in the guinea-pig colon. Am. J. Physiol. 264:G74-G80.

33. North, R.A., and Surprenant, A. 1985. Inhibitory synaptic potentials resulting from alpha 2 adrenoceptor activation in guinea-pig submucous plexus neurones. J. Physiol. (Lond.) 358:17-33.

34. Wood, J.D. 1994. Physiology of the enteric nervous system. In Physiology of the gastrointestinal tract. L.R. Johnson, D.H. Alpers, J. Christensen, E.D. Jacobson, and J.H. Walsh, editors. Raven Press. New York, NY. 423-482.

35. Lin, J.Y., and Krier, J. 1995. Human recombinant interleukin-1 beta inhibits nicotinic transmission in neurons of guinea pig pelvic plexus ganglia. Am. J. Physiol. 32:G981-G987.

36. Yu, B.J., and Shinnick-Gallagher, P. 1994. Interleukin-1 beta inhibits synaptic transmission and induces membrane hyperpolarization in amygdala neurons. J. Pharmacol. Exp. Ther. 271:590-600.

37. Cooke H.J., and Reddix, R.A. 1994. Neural regulation of intestinal electrolyte transport. In Physiology of the gastrointestinal tract. L.R. Johnson, D.H. Alpers, J. Christensen, E.D. Jacobson, and J.H. Walsh, editors. Raven Press. New York, NY. 2083-2132.

38. Wood, J.D. 1982. The synaptic interface between the sympathetic and enteric divisions of the autonomic nervous system. In Functional bowel disorders. W. Chey, editor. Raven Press. New York, NY. 87-91.

39. Manber, L., and Gershon, M.D. 1979. A reciprocal adrenergic-cholinergic axoaxonic synapse in the mammalian gut. Am. J. Physiol. 5:E738-E745.

40. Nishi, S., and North, R.A. 1973. Intracellular recording from the myenteric plexus of the guinea-pig ileum. J. Physiol. (Lond.) 231:471-491.

41. Hirst, G.D.S., and McKirdy, H.C. 1974. Presynaptic inhibition at a mammalian peripheral synapse. Nature. 250:430-431. 